\title{
A solution to linear programming problems with type 2 fuzzy parameters
}

\author{
Anny M. A. Silva ${ }^{1}$ \\ IFPR, Pinhais, PR \\ Akebo Yamakami \\ FEEC/UNICAMP, Campinas, SP
}

\begin{abstract}
In this work, a new solution proposal for linear programming problems will be presented, whose parameters are type 2 fuzzy sets. For this, we will use the methods proposed by Zimmermann in [9] and by Figueroa in [2].
\end{abstract}

Keywords. Fuzzy, Type 2 Fuzzy Sets, Fuzzy Restrictions.

\section{Introduction}

In this work, we will purpose two theories widely used in the resolution of a class of problems: Fuzzy Theory and Optimization (linear programming). These two theories are relate in various aspects and have the ability to solve/model real problems. Linear programming uses mathematical modeling to describe a problem at be treated looking for an optimal result for these problems, that is, a solution that satisfies, in the best possible way, a certain objective.

Linear programming problems do not always involve precise elements, and in many cases during the modeling process, we are faced with uncertain data or information. Thus, some ways to incorporate these defined uncertainties to be used in solve problems. One of the ways to deal with these information is to make use of the theory of fuzzy sets that was presented to us by Zadeh in 1965. In a previous work [1], Bellman and Zadeh used the concepts of Fuzzy sets to formulate decision-making strategies in Fuzzy environments.

In this work we will use the concept of type 2 fuzzy sets [5]. This theory has become a widely researched area in recent years. It was introduced in 1975 by Zadeh [8], as an extension of type 1 fuzzy sets. The main difference between a type 1 fuzzy set and a type 2 fuzzy set is that the degree of relevance of a type 1 fuzzy set is a crisp number, while the degree of relevance of type 2 fuzzy set is the type 1 fuzzy set (see [7]). These sets allow us to treat uncertainties about linear programming problems in another way.

Based on papers presented in [2] and [9], we propose a new approach to solve linear programming problems with type 2 fuzzy interval restrictions, seeking to improve the results obtained by Figueroa in his works [2]. This work is an extension of the method proposed in [6], as studies of linear programming problems with fuzzy parameters type 2 were initiated by the authors. The proposed method is based on the Zimmermann method (see [9], [10], [11]) and also on the Figueroa method in [2]. It starts from the principle of reducing the type 2 fuzzy interval set to a type 1 fuzzy set while maintaining the non-linearity obtained with the defuzzification.

\footnotetext{
1 anny.silva@ifpr.edu.br

2akebo@dt.fee.unicamp.br
} 


\section{Type 2 Fuzzy Sets}

In this section we presented some important definitions for the development of this work. We are considering the concepts of fuzzy sets type 2 and type 2 interval. These concepts are important because when working with decision-making problems we find many uncertainties about the data to be treated and these concepts allow us to obtain a greater degree of freedom to model these uncertainties. Many of the definitions that is presented bellow can be seen in [3], [4] and [5].

Definition 1. A type 2 fuzzy set, denoted $\tilde{A}$, is a function on the Cartesian product

$$
\begin{gathered}
\varphi: X \times[0,1] \rightarrow[0,1] \\
(x, u) \rightarrow \varphi_{\tilde{A}}(x, u),
\end{gathered}
$$

where $U$ is the universe for the primary variable of $\tilde{A}$, x. The $3 \mathrm{D}$ membership function of $\tilde{A}$ is usually denoted $\varphi_{\tilde{A}}(x, u)$, where $x \in X$ and $u \in U=[0,1]$, that is,

$$
\tilde{A}=\left\{\left((x, u), \varphi_{\tilde{A}}(x, u)\right) \mid x \in X \times[0,1], \varphi_{\tilde{A}}(x, u) \in[0,1]\right\},
$$

in which $0 \leq \varphi_{\tilde{A}}(x, u) \leq 1$.

$\tilde{A}$ can also be expressed as

$$
\tilde{A}=\int_{x \in X} \int_{u \in[0,1]} \frac{\varphi_{\tilde{A}}(x, u)}{(x, u)}
$$

where $\iint$ denotes union over all admissible $x$ and $u$. For discrete universes of discourse $\int$ is replaced by $\sum$, and $X$ and $U$ by $X_{d}$ and $U_{d}$.

In Definition 1 , the first constraint $\forall u \in[0,1]$ is equivalent to constraint of a type 1 fuzzy set, $0 \leq \varphi_{A}(x) \leq 1$. So when the uncertainties disappear, the type 2 membership role becomes a type 1 membership role. The second restriction, $0 \leq \varphi_{\tilde{A}}(x, u) \leq 1$ is consistent with the fact that the readth of membership functions should take values between 0 and 1 .

In Eqs. (1) and (2) $u$ is called the secondary variable and has domain $U=[0,1]$ at each $x \in X$.

Definition 2. When $\varphi_{\tilde{A}}(x, u)=1$ for $\forall x \in X$ and $\forall u \in U$, then $\tilde{A}$ called an interval type-2 fuzzy set.

Although the third dimension of the type 2 fuzzy set is no longer needed because it conveys no new information about the interval type 2 fuzzy set, the interval type 2 fuzzy set can still be expressed as a special case of the type 2 fuzzy set as:

$$
\tilde{A}=\int_{x \in X} \int_{u \in[0,1]} \frac{1}{(x, u)} .
$$

Definition 3. At each value of $x$, say $x=x^{\prime}$ the $2 \mathrm{D}$ plane whose axes are $u$ and $\varphi_{\tilde{A}\left(x^{\prime}, u\right)}$ is called a vertical slice of $\varphi_{\tilde{A}\left(x^{\prime}, u\right)}$. A secondary membership function is a vertical slice of $\varphi_{\tilde{A}(x, u)}$. It is $\varphi_{\tilde{A}\left(x=x^{\prime}, u\right)}$ for $x^{\prime} \in X$ and $\forall u \in[0,1]$, that is,

$$
\varphi_{\tilde{A}\left(x=x^{\prime}, u\right)} \equiv \varphi_{\tilde{A}\left(x^{\prime}\right)}=\int_{u \in J_{x^{\prime}}^{u} \subseteq[0,1]} \frac{1}{u}
$$

where $J_{x^{\prime}}^{u}$ is the subset of $U$ that is the support of $\varphi_{\tilde{A}}\left(x^{\prime}\right)$ and is called the primary membership of $\tilde{A}$. If $J_{x}^{u}$ is connected, then it can be represented by

$$
J_{x}^{u}=\left\{(x, u) \mid u \in\left[\underline{\varphi}_{\tilde{A}}(x), \bar{\varphi}_{\tilde{A}}(x)\right]\right\} .
$$


The amplitude of the secondary membership function is called the secondary grade and for a interval type 2 fuzzy set, all secondary grades are equal to 1 . Because $\forall x^{\prime} \in X$, we drop the prime notation on $\varphi_{\tilde{A}}\left(x^{\prime}\right)$ and refer to $\varphi_{\tilde{A}}(x)$ a secondary membership function; it is the membership function of a type 1 fuzzy set, which we also refer to as a secondary set, $\tilde{A}(x)$.

Definition 4. The upper membership function (UMF) and lower membership function (LMF) of $\tilde{A}$ are two type 1 membership function that bound $F O U(\tilde{A})$. The UMF is associated with the upper bound of $\operatorname{FOU}(\tilde{A})$ and is denoted $\bar{\varphi}_{\tilde{A}}(x), \forall x \in X$ and the LMF is associated with the lower bound of $\operatorname{FOU}(\tilde{A})$ and is denoted $\underline{\varphi}_{\tilde{A}}(x)$

$$
\begin{aligned}
& \bar{\varphi}_{\tilde{A}}(x)=\sup \left\{u \mid u \in[0,1], \varphi_{\tilde{A}}(x, u)>0\right\} \forall x \in X \\
& \underline{\varphi}_{\tilde{A}}(x)=\inf \left\{u \mid u \in[0,1], \varphi_{\tilde{A}}(x, u)>0\right\} \forall x \in X
\end{aligned}
$$

Definition 5. The uncertainty domain (DOU) for a fuzzy set of type 2 is a union of sets $J_{x}^{u}$, given by

$$
\operatorname{DOU}(\tilde{A})=\bigcup_{x \in X} J_{x}^{u}
$$

These sets represent the union of all primary memberships.

Definition 6. Uncertainty in the primary memberships of an interval type 2 fuzzy set, $\tilde{A}$, consists of a bounded region that is called the footprint of uncertainty (FOU). The FOU is the DOU for connected domains, it is the two-dimensional support of $\tilde{A}$, that is,

$$
F O U(\tilde{A})=\left\{(x, u) \mid x \in X, u \in\left[\underline{\varphi}_{\tilde{A}}(x), \bar{\varphi}_{\tilde{A}}(x)\right]\right\} .
$$

This is a vertical-slice representation of the FOU, because each of the primary memberships is the support of a vertical slice. Mathematically the FOU is the union of all the primary membership functions in which for the discrete universe $X \times U$ is just a collection of points.

The secondary grades of an interval type 2 fuzzy set convey no new information, the FOU is a complete description of an interval type 2 fuzzy set. The uniformly shaded FOU of an interval type 2 fuzzy set denotes that there is a uniform distribution that sits on top of it.

We can see the representation of some Type 2 Fuzzy Sets in Figure 1.

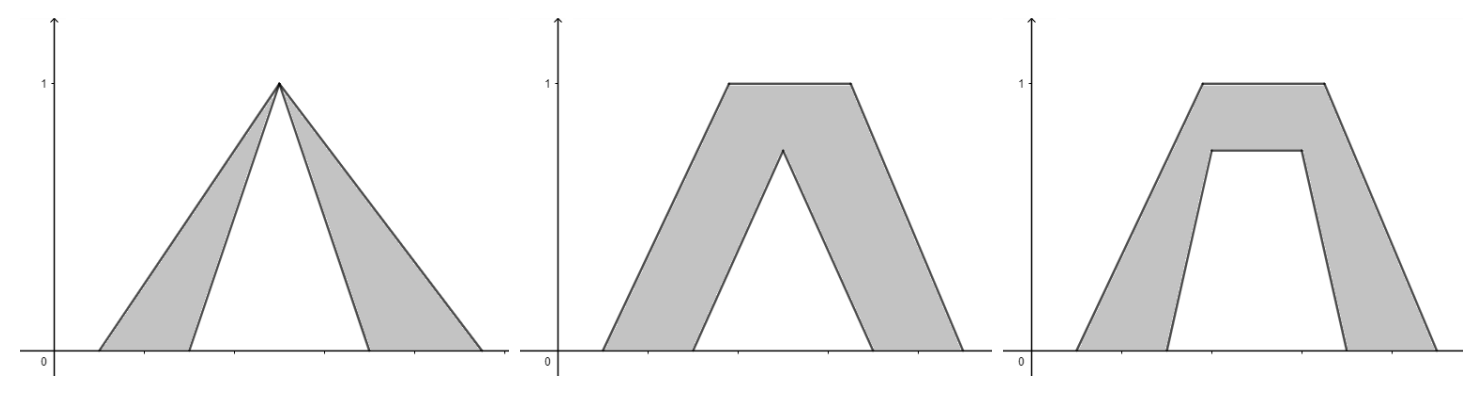

(a)

(b)

(c)

Figure 1: Representation of type 2 fuzzy sets 


\section{Linear fuzzy programming with type 2 fuzzy restrictions}

A type 2 fuzzy linear programming problem is solved using infinite membership functions that are involved in the FOU of that set. Our proposal is also based on the principle of reducing a type 2 fuzzy problem in a type 1 fuzzy problem, calculating, simultaneously, values $b_{l}^{k}$ and $b_{r}^{k}$ that are inside the FOU and are, respectively, between $\underline{b}_{1}^{k}$ and $\bar{b}_{1}^{k}$ and $\underline{b}_{2}^{k}$ and $\bar{b}_{2}^{k}$.

Optimal values are found within the FOU. The initial problem can be represented as follows:

$$
\begin{aligned}
& z=\max c x \\
& \text { subject to } \\
& \begin{aligned}
A x & \precsim \tilde{b}, \\
x & \geq 0
\end{aligned}
\end{aligned}
$$

where $x, c \in \mathbb{R}^{m}, A \in \mathbb{R}^{m \times n}, \tilde{b}$ is an interval type 2 fuzzy set vector defined by two primary membership functions $\underline{\varphi}_{\tilde{b}}$ and $\bar{\varphi}_{\tilde{b}}$ and $\precsim$ is a Type 2 fuzzy partial order. The pertinence functions of the problem are given by:

$$
\underline{\varphi}_{\tilde{b}}=\left\{\begin{array}{l}
1, \text { if } \quad x \leq \underline{b}_{1}^{k} \\
\frac{\underline{b}_{2}^{k}-x}{\underline{b}_{2}^{k}-\underline{b}_{1}^{k}}, \text { if } \underline{b}_{1}^{k} \leq x \leq \underline{b}_{2}^{k} \\
0, \text { if } x \geq \underline{b}_{2}^{k}
\end{array} \quad \text { and } \bar{\varphi}_{\tilde{b}}=\left\{\begin{array}{l}
1, \text { if } x \leq \bar{b}_{1}^{k} \\
\frac{\bar{b}_{2}^{k}-x}{\bar{b}_{2}^{k}-\bar{b}_{1}^{k}}, \text { if } \bar{b}_{1}^{k} \leq x \leq \bar{b}_{2}^{k} \\
0, \text { if } \quad x \geq \bar{b}_{2}^{k}
\end{array},\right.\right.
$$

In Figure 2 we can see the representation of the type 2 fuzzy set.

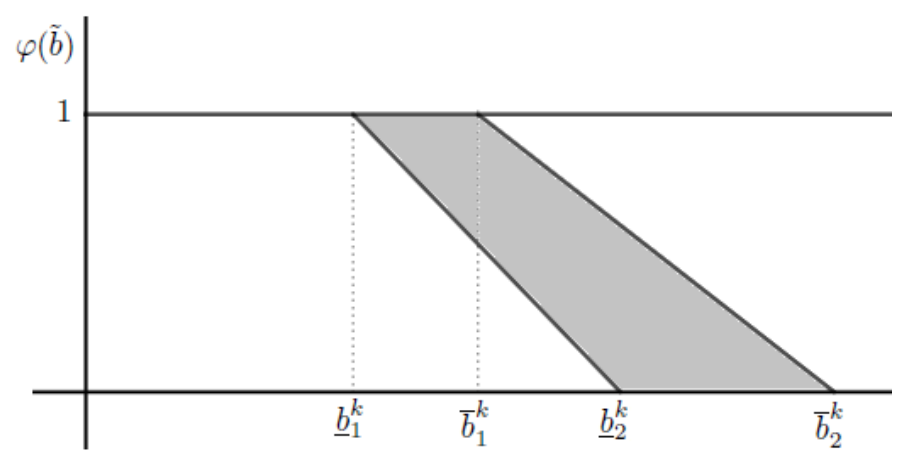

Figure 2: type 2 fuzzy set.

To solve the problem (3), we calculate simultaneously, within the FOU the values:

$$
\underline{b}^{k}=\left(1-\lambda_{l}^{k}\right) \underline{\underline{b}}_{1}^{k}+\lambda_{l}^{k} \bar{b}_{1}^{k} \quad \text { and } \quad \bar{b}^{k}=\left(1-\lambda_{r}^{k}\right) \underline{b}_{2}^{k}+\lambda_{r}^{k} \bar{b}_{2}^{k}
$$

with $\underline{b}_{1}^{k} \leq b_{l}^{k} \leq \bar{b}_{1}^{k}, \underline{b}_{2}^{k} \leq b_{r}^{k} \leq \bar{b}_{2}^{k} k$ and $0 \leq \lambda_{r}^{k}, \lambda_{l}^{k} \leq 1$. 
So we have the following problem:

$$
\begin{gathered}
\max \alpha \\
\text { s.a } \\
c x-\alpha\left(z_{r}^{k}-z_{l}^{k}\right) \geq z_{l}^{k} \\
A x \leq(1-\alpha)\left[\left(1-\lambda_{r}^{k}\right) \underline{b}_{2}^{k}+\lambda_{r}^{k} \bar{b}_{2}^{k}\right]+\alpha\left[\left(1-\lambda_{l}^{k}\right) \underline{b}_{1}^{k}+\lambda_{l}^{k} \bar{b}_{1}^{k}\right] \\
x \geq 0, \alpha \in[0,1]
\end{gathered}
$$

where $\alpha, \lambda_{r}^{k}$ and $\lambda_{l}^{k}$ are unknown, we have a problem non-linear in (4).

To linearize the problem above, we will make the following substitutions

$$
\alpha \lambda_{l}^{k}=D_{1} \quad \text { and } \quad(1-\alpha) \lambda_{r}^{k}=D_{2} .
$$

So, we come to a equivalent problem:

$$
\begin{gathered}
\max \alpha \\
\text { s.a } \\
{[A x]^{k} \leq\left(\alpha I_{m}-D_{1}\right) \underline{b}_{1}^{k}+D_{1} \bar{b}_{1}^{k}+\left((1-\alpha) I_{m}-D_{2}\right) \underline{b}_{2}^{k}+D_{2} \bar{b}_{2}^{k}} \\
\alpha I_{m}-D_{1} \geq 0 \\
(1-\alpha) I_{m}-D_{2} \geq 0
\end{gathered}
$$

Where $A \in \mathbb{R}^{m x n}, b \in \mathbb{R}^{m}, x \in \mathbb{R}^{n}, z_{r}^{k}$ is an aspiration level of the decision maker, $z_{r}^{k}-z_{l}^{k}$ is the degree of admissible of violation of restrictions. and $I_{m}$ is an identity matrix and $D_{i}, i: 1,2$, are diagonal matrices.

\section{Computational tests}

Example 1. Now, let's compare our method using the example given by Figueroa in [2], where the main idea is to compute $z^{*}=c\left(x^{*}\right)$. All parameters of $\tilde{b}, c$ and $A$ are defined in matrix form.

$$
\begin{aligned}
& A=\left[\begin{array}{ccc}
5 & 3 & 7 \\
10 & 4 & 9 \\
4 & 6 & 3 \\
2 & 7 & 7 \\
5 & 6 & 11
\end{array}\right] ; c=\left[\begin{array}{l}
12 \\
17 \\
19
\end{array}\right] \\
& \underline{b}_{1}=\left[\begin{array}{c}
50 \\
70 \\
40 \\
60 \\
40
\end{array}\right] ; \bar{b}_{1}=\left[\begin{array}{c}
72 \\
104 \\
65 \\
95 \\
80
\end{array}\right] ; \underline{b}_{2}=\left[\begin{array}{c}
60 \\
80 \\
55 \\
75 \\
57
\end{array}\right] ; \bar{b}_{2}=\left[\begin{array}{c}
95 \\
110 \\
77 \\
102 \\
98
\end{array}\right]
\end{aligned}
$$

With the method proposed in [2], Figueroa obtains the following results: $\underline{z}_{1}=113.33, \bar{z}_{1}=$ 157.16, $\underline{z}_{2}=189.68, \bar{z}_{2}=223.5$. In [2], the values obtained for $z_{l}$ and $z_{r}$ are, respectively $z_{l}=135.25, z_{r}=206.59, \alpha^{*}=0.511$ and $z^{*}=171.55$.

With our method, using the values of $\bar{b}_{1}^{k}$ and $\bar{b}_{2}^{k}$ obtained were as following: $z_{l}=157.16$, $z_{r}=223.25, \alpha^{*}=0.5$ and $z^{*}=190.33$. In this example, the value we obtained for $\alpha^{*}$ was very close to the value obtained in [2] and the value obtained for $z^{*}$, was better than the value in [2].

Figure 3 illustrates the results obtained for the example. 


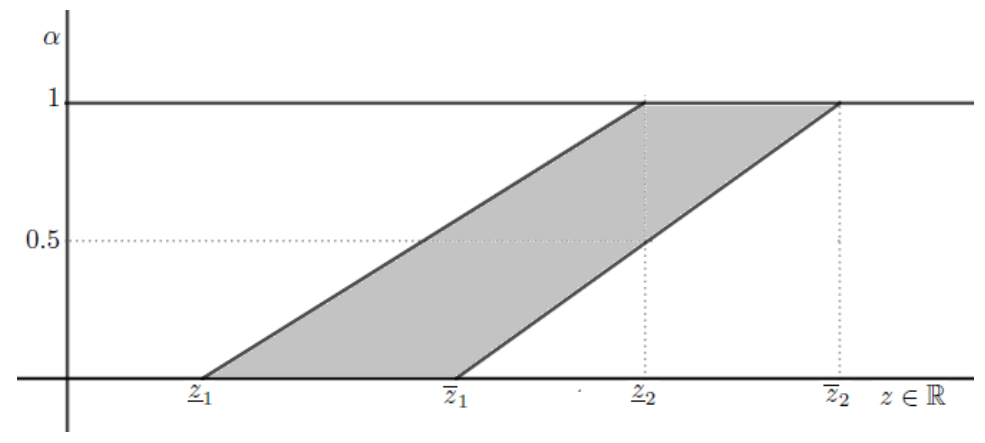

Figure 3: Results obtained in your model: $\underline{z}_{1}=113.33, z_{l}=\bar{z}_{1}=157.16, z_{r}=\underline{z}_{2}=189.68$, $\bar{z}_{2}=223.5$.

In example we obtain approximate solutions to the proposed problem and these solutions are better than those found in [2]. Thus, we can say that, with regard to the solution found, the new method obtains better results.

\section{Conclusion}

In this paper a new method to solve linear programming problems with type 2 fuzzy interval constraints has been proposed. The method used by Zimmermann [9] for type 1 linear fuzzy programming problems and the method proposed by Figueroa [2] for type 2 interval fuzzy linear programming problems were used for the development of this method.

The main difference between method presented in [2] and the method proposed in this work if is with regard to the results obtained. With the method proposed, the treatment of the problem data provides us with solutions that tend to to be more efficient than that of the other methods, and that gives us a better scope to work with the possible errors of the problems.

\section{Acnowledgments}

Anny M. A. Silva thanks CAPES for the financial support during the first years of the Doctorate.

\section{References}

[1] Bellman, R. and Zadeh, L. A., Decision making in a fuzzy environment, Management Sci, 17: 141-164, 1970.

[2] Figueroa, J. C. and Hernández, G., Computing optimal solutions of a Linear programming problem with interval type-2 fuzzy constraints. Lecture Notes in Computer Science, 7208: 567-576, 2012.

[3] Jafelice, R.S.M., Cabrera, N.V., Câmara, M.A., Sistemas p-fuzzy utilizando conjuntos fuzzy do tipo 2 intervalar. Biomatemática - UNICAMP, 28: 1-14, 2018.

[4] Mendel, J. M., Advances in type-2 fuzzy sets and systems, Information sciences, 177: 84-110, 2007. 
[5] Mendel, J. M., Introduction to type-2 fuzzy logic control, Theory and applications. IEEE, New Jersey, 2014.

[6] Silva, A. M. A. and Yamakami, A. Modelo de Programação Linear Fuzzy Tipo 2 Intervalar, $V$ Congresso Brasileiro de Sistemas Fuzzy, 2018.

[7] Zadeh, L. A., Information and control, Fuzzy sets, World Scientific, 8: 338-353, 1965.

[8] Zadeh, L.A., The concept of a linguistic variable and its application to approximate reasoning1, Informat. Sci, 8: 199-249, 1975.

[9] Zimmermann, H.-J., Description and optimization of fuzzy systems, International Journal General Systems, 2: 209-215, 1976.

[10] Zimmermann, H.-J., Fuzzy programming and linear programming with several objective functions, Fuzzy Sets and Systems, 1: 45-56, 1978.

[11] Zimmermann, H.-J., Fuzzy mathematical programming, Computers \& Operations Research, 10: 291-298, 1983 